This is the peer reviewed version of the following article: "Rheological and physical characterization of PEDOT: PSS/graphene oxide nanocomposites for perovskite solar cells", which has been published in final form at [http://onlinelibrary.wiley.com/doi/10.1002/pen.24554/abstract]. This article may be used for non-commercial purposes in accordance with Wiley Terms and Conditions for Self-Archiving."

Rheological and physical characterization of PEDOT: PSS/graphene oxide nanocomposites for perovskite solar cells

Antonella Giuri, Sofia Masi, Silvia Colella, Andrea Listorti, Aurora Rizzo, Alessandro Kotuvn, Simone Dell'Elce, Andrea Liscio, Carola Esposito Corcione

A. Giuri, Dr. C. Esposito Corcione

Dipartimento di Ingegneria dell'Innovazione, Università del Salento, via per Monteroni, km 1, 73100, Lecce, Italy

Dr. S. Masi, Dr. A. Rizzo

Istituto di Nanotecnologia CNR-Nanotec, Polo di Nanotecnologia c/o Campus Ecotekne, via Monteroni, 73100 Lecce, Italy

Dr. S. Colella, Dr. A. Listorti 
Dipartimento di Matematica e Fisica “E. De Giorgi”, Università del Salento, Via Arnesano snc, 73100 Lecce, Italy

A. Kovtun, S. Dell'Elce, Dr. A. Liscio

Istituto per la Sintesi Organica e la Fotoreattività CNR-ISOF, via Gobetti 101, 40120, Bologna, Italy

Dr. A. Liscio

Istituto dei Sistemi Complessi CNR-ISC, via del Fosso del Cavaliere 100, 00133, Roma, Italy 


\section{Rheological and physical characterization of PEDOT: PSS/graphene oxide nanocomposites for perovskite solar cells}

Antonella Giuri ${ }^{1}$, Sofia Masi ${ }^{2}$, Silvia Colella ${ }^{2,3}$, Andrea Listorti ${ }^{2,3}$, Aurora Rizzo ${ }^{2}$, Alessandro Kotuvn $^{4}$, Simone Dell'Elce ${ }^{4}$,Andrea Liscio ${ }^{4,5}$, Carola Esposito Corcione $^{1}$

\footnotetext{
${ }^{1}$ Dipartimento di Ingegneria dell'Innovazione, Università del Salento, via per Monteroni, km 1, 73100, Lecce, Italy

${ }^{2}$ Istituto di Nanotecnologia CNR-Nanotec, Polo di Nanotecnologia c/o Campus Ecotekne, via Monteroni, 73100 Lecce, Italy

${ }^{3}$ Dipartimento di Matematica e Fisica “E. De Giorgi”, Università del Salento, Via Arnesano snc, 73100 Lecce, Italy

${ }^{4}$ Istituto per la Sintesi Organica e la Fotoreattività CNR-ISOF, via Gobetti 101, 40120, Bologna, Italy

${ }^{5}$ Istituto dei Sistemi Complessi CNR-ISC, via del Fosso del Cavaliere 100, 00133, Roma, Italy
}

\section{ABSTRACT}

In this work, the influence of graphene oxide (GO) doped Poly(3,4 ethylenedioxythiophene):poly (styrenesulfonate)(PEDOT:PSS) thin nanocomposite on an indium-tin-oxide (ITO) anode, as hole transport layer (HTL) in perovskite solar cells, was investigated. Different concentrations of GO were added into the PEDOT:PSS in order to enhance its conductivity. In particular, the influence of GO content on the rheological and thermal properties of Poly(3,4ethylenedioxythiophene):poly(styrenesulfonate) (PEDOT:PSS)/GO nanocomposites was initially examined. The GO filler was prepared by using modified Hummers method and dispersed into PEDOT:PSS in different quantity (ranging from 0.05 to $0.25 \% \mathrm{wt} / \mathrm{wt}$ ). The obtained nanocomposite solutions were analyzed by rheological characterizations in order to evaluate the influence of the GO filler on the viscosity of the PEDOT:PSS matrix. The wettability of solutions was evaluated by Contact Angle (CA) measurements. The quality of GO dispersion into the polymer matrix was studied using Scanning electron microscopy (SEM) and X-ray diffraction (XRD). Thermal characterizations (DSC and TGA) were, finally, applied on nanocomposite films in order to evaluate thermal stability of the films as well as to indirectly comprehend the GO influence on PEDOT:PSS-water links. 


\section{INTRODUCTION}

Since the discovery of conductive polyacetylene in 1978 [1-3], conductive polymers have attracted the attention worldwide because they combine the properties of metals and polymers [4]. However, application and processability of the conducting polymers are not always easy to balance [5]. Poly(3,4-ethylenedioxythiophene):poly(styrenesulfonate) (PEDOT:PSS) is one of the most successful conjugated polymers being used commercially as charge extractor since they were synthesized in the late 1980s [5]. PEDOT:PSS films can be readily deposited on substrates by the conventional solution processing techniques [6,7]. PEDOT:PSS is a polyelectrolyte with the hydrophobic PEDOT and hydrophilic PSS, among which a Coulombic interaction exists: PSS of PSS anions' segments attached with PEDOT are screened by the positive charges on PEDOT. Moreover, the PSS segments form blobs for preventing the contact of PEDOT from water in order to reduce the interactions between PEDOT and water. The blobs have a core/shell structure with PEDOT in the core and PSS in the shell $[4,8]$.

To date, the solid polyelectrolyte PEDOT-PSS was the most common organic charge extraction layer used in polymer solar cells. It allows efficient charge extraction and transport, but suffers important drawbacks related to the stability of the PEDOT:PSS itself [9-11]. Aiming at enhancing the electrical conductivity and the stability of polymeric matrices for photovoltaic applications, the doping of PEDOT:PSS by graphene based filler has attracted great attention and has shown interesting results [12-16]. The authors have already analyzed the influence of GO on electrical conductivity of PEDOT:PSS as reported in previous study [16]. In details, the electrical properties of the PEDOT:PSS/GO film after the annealing was studied by DC electrical measurements with fourpoints probe. The sheet resistance measured on pure PEDOT:PSS film amounts $50 \mathrm{M} \Omega \square-1$, in agreement with literature [17]. After adding the lowest amount of GO (0.05\%wt), the resistance decreases down to $180 \mathrm{k} \Omega \square-1[16]$. 
Graphene, a two-dimensional carbon layer, shows, in fact, interesting thermal, elastic, optical, electrical, and mechanical properties. Despite the extraordinary properties, the graphene production, including chemical vapor deposition (CVD), mechanical exfoliation, epitaxial growth [18], requires expensive, complex and not often compatible with upscaling processes. An alternative and promising approach to produce large amount of solution processable graphene is the reduction of graphene oxide (GO) dispersions. Prepared by modified Hummer's method [19,20], GO is a single layer graphenebased material presenting different chemical functional groups such as oxygen epoxides (bridging oxygen atoms), carbonyls $(\mathrm{C}=\mathrm{O})$, hydroxyls $(-\mathrm{OH})$ and phenols. Forming stable solutions in water, GO is high-processable, flexible, and low-cost material, having remarkable potential applications for consumer electronics. The disrupted $\mathrm{sp} 2$ conjugation of the graphene lattice leads to a deterioration of conductivity, but its residual sp2 clusters can still allow hole transportation to occur via hopping [21]. The electrical conductivity can be recovered by reducing GO, removing the oxygen-contained in the functional groups. Thermal and chemical approaches are typically used to reduce GO, exploiting high temperature (up to $900^{\circ} \mathrm{C}$ ) or toxic chemical agent (i.e. hydrazine). Green approaches such as UV irradiation [22-24]or glucose [25] as reduction agents, are recently presented showing interesting results in term of reduction efficiency $[15,16,18,22,24,26]$.

We recently demonstrated the improvement of the performance of photovoltaic devices by blending PEDOT:PSS and reduced graphene oxide (RGO) as thin film electrode $[15,16]$. The change of the surface wettability improves the homogeneity of the upper PEDOT:PSS layer while the presence of reduced GO sheets restores and improves the electrical conductibility of the film. $[15,16]$. However the dependence of macroscopic electrical properties on the evolution of the microstructure of doped PEDOT:PSS is not clearly understood. Zhou et al. firstly investigated the special arrangement of the ordered and disordered phases in PEDOT:PSS nanofilms clarifying how amorphous PSS hinders electrical transport at various length scales in the PEDOT:PSS films [27].

The understanding of the role of GO into the PEDOT:PSS matrix is crucial to simplify the process of the film production and as well as to improve the performance of the device. In this work, we report 
a detailed investigation of the influence of GO on the rheological, morphological and thermal properties of the composite.

\section{Experimental}

\section{Materials}

PEDOT:PSS aqueous solution (Clevios PVP AI4083) was purchased from Heraeus with a PEDOT:PSS concentration of $1.3 \% \mathrm{w} / \mathrm{w}$ (weight ratio of PSS to PEDOT =6).

GO was prepared using modified Hummers method [19] yielding GO flakes with maximum lateral size of $100 \mu \mathrm{m}[20]$.

\section{GO/PEDOT:PSS nanocomposite preparation}

The dispersion of GO into PEDOT:PSS was obtained by the solvent swelling method [15,16,28]. GO was added into PEDOT:PSS and the blend was magnetically stirred for 90' and sonicated for $15^{\prime}$ at room temperature (RT). The influence of each component was studied by preparing different concentrations of GO/PEDOT:PSS dispersions:(0.05, 0.15, 0.25 \%wt/wt) (Table 1). The blend was deposited at RT in air on different substrates in accordance with the type of characterization performed.

\section{Experimental techniques}

The rheological characterization of the all formulations produced was carried out in a strain controlled rheometer (Ares TA Instrument). Viscosity was measured at $20.5^{\circ} \mathrm{C}$ using a parallel plate geometry (radius $=12.5 \mathrm{~mm}$ ) in steady state mode with a shear rate ranging from 0.05 to $600 \mathrm{~s}^{-1}$. A pre-shear experiment was run prior to each test, and the rheological experiments were repeated at least three times for checking the repeatability of results. At the end of the rheological characterization, an appropriate theoretical equation was identified for each system in order to fit the experimental data. 
GO/PEDOT:PSS dispersions were drop casted on a glass substrate and analyzed, after water evaporation, by XRD in order to evaluate the quality of GO dispersion into PEDOT:PSS. The XRD (Rigaku, Tokyo, Japan) pattern was obtained with $\mathrm{CuK} \alpha$ radiation $\left(\lambda=1.5418 \mathrm{~A}^{\circ}\right)$ in the step scanning mode recorded in the $2 \theta$ range of $5^{\circ}-50^{\circ}$, with a step size of $0.02^{\circ}$ and step duration of $0.5 \mathrm{~s}$.

GO/PEDOT:PSS solutions were spin coated on ITO substrates at $2500 \mathrm{rpm}$ for 60 ” and annealed at $140^{\circ} \mathrm{C}$ for $1 \mathrm{~h}$ in $\mathrm{N}_{2}$ atmosphere in order to analyze the morphology of the films obtained by SEM, and in particular to evaluate the quality of various GO dispersions into PEDOT:PSS.

SEM micrographs were obtained using Carl Zeiss Auriga40 Crossbeam instrument, in high vacuum and high-resolution acquisition mode, equipped with Gemini column and an integrated high efficiency in-lens detector. The applied acceleration voltage was 2 or $5 \mathrm{kV}$.

The thermal stability of pristine PEDOT:PSS and GO/PEDOT:PSS was investigated by thermogravimetric analyses, carried out on a TGA/DSC 1 manufactured by Mettler Toledo, under a nitrogen atmosphere created by fluxing $50 \mathrm{ml} / \mathrm{min}$ of $\mathrm{N}_{2}$ from $20^{\circ} \mathrm{C}$ to $600^{\circ} \mathrm{C}$ at the heating rate of $10^{\circ} \mathrm{C} / \mathrm{min}$. About $5 \mathrm{mg}$ of sample was put into alumina pan for the test after air drying for 96 hours samples drop casted on cleaned silicone sheet [29]. The results obtained from TGA in the range of $20-160^{\circ} \mathrm{C}$ were combined with the analysis by a differential scanning calorimeter (DSC Mettler Toledo 622) in order to comprehend the reaction that occurs in all the systems. About $5 \mathrm{mg}$ of dried samples for 96 hours on silicone sheet were put into aluminum flat disks and heated from 20 up to $160^{\circ} \mathrm{C}$ at $10^{\circ} \mathrm{C} / \mathrm{min}$ under nitrogen atmosphere flow at $80 \mathrm{~mL} / \mathrm{min}$. After free cooling up to room temperature, a second heating scan was performed.

The wettability of PEDOT:PSS and GO/PEDOT:PSS blends on ITO substrates was evaluated by contact angle (CA) measurements with a First Ten Angstroms FTA1000 Quick start instrument. The nanocomposite dispersions were spin coated on a quartz substrate and analyzed by UV-visible spectrophotometer (Varian Cary 500) after thermal annealing in order to evaluate the influence of GO concentration on film absorption. The Ultraviolet-visible absorption spectra were recorded in the 200-800 nm wavelength range at room temperature. 


\section{Results and discussion}

The viscosity of neat PEDOT:PSS as a function of the shear rate (ranging from 0.05 to $600 \mathrm{~s}^{-1}$ ) was compared with that of PEDOT:PSS filled with different GO contents $(0.05,0.15$ and $0.25 \%$ wt/wt $)$ and reported in Figure 1(a). A pseudo-plastic behavior was evidenced for each mixture investigated. After adding GO, the viscosity of the PEDOT:PSS increases, probably due to the interaction between the functional groups of GO and PEDOT:PSS. In order to estimate the value of the viscosity reached during the film deposition (by spin coating at $3000 \mathrm{rpm}$ ), the shear rate reached during the spin coating process was calculated by using the following equation:

$$
=\frac{v}{h}
$$

Where, $v$ is the tangential velocity (equal to the product of angular spin coating velocity and the radius of rheometer plate) and $h$ is the sample thickness (i.e. the distance between the plates among which there is the solution).

The shear rate calculated, starting from eq. (1), is $9817.5 \mathrm{~s}^{-1}$, which is out of the measurement range of the available rheometer (i.e. $600 \mathrm{~s}^{-1}$ ). For this reason, proper theoretical models were applied to fit the experimental data, reported in Figure 1(a). In details, Cross [30] proposed a model allowing to predict the plateau values for lower and upper Newtonian viscosities, indicated as ${ }_{0}$ and , respectively:

$$
\eta=\eta_{\infty}+\frac{\eta_{0}-\eta_{\infty}}{1+(\tau \dot{\gamma})^{m}}
$$

where, $\tau$ and $\mathrm{m}$ are model parameters and $\dot{\gamma}$ the shear rate.

In particular, $\tau$ corresponds to the reciprocal of the shear rate at which the calculated value of

equals ${ }_{0}$, while the parameter $\mathrm{m}$ is related to the power low index, $\mathrm{n}$, by the expression: $\mathrm{m}=1-\mathrm{n}$. The values of the parameters in eq. (2), calculated by a non linear fitting of the experimental data shown 
in Figure 1(a), are reported in Table 2. At very low shear rates, the viscosity of GO/PEDOT mixtures is considerably higher than that of pure PEDOT:PSS, as already pointed out. In particular, $\mathrm{GO}_{0.25} \mathrm{PEDOT}$ formulation possesses the highest viscosity. At higher shear rate values (i.e., ), the viscosity of the mixtures is also higher than that of pure PEDOT:PSS, especially in the presence of 0.15 and $0.25 \%$ wt of GO. The values of the parameter $\mathrm{m}$, calculated for the mixtures containing GO, are very similar and different from that of neat PEDOT:PSS, as witnessed by their rheological curves. On the other hand, the values of $\tau$ increases by increasing the content of GO, indicating that the pseudo-plastic behavior starts at lower shear rate values for the nanocomposite mixtures.

The experimental data of Figure 1 (a) was fitted (at high shear rate), by using Batchelor equation [31], obtained from the correction of the classical Einstein model, in order to take into account the effect of more concentrated suspensions of particles $(\varphi>0.02)$ [32-34]:

$$
\eta_{r}=\frac{\eta_{\infty}}{\eta_{\infty s}}=1+k_{1} \cdot \varphi+k_{2} \cdot \varphi^{2}
$$

where, is the viscosity of the GO/PEDOT solutions at high shear rate, and ${ }_{s}$ the viscosity of the suspending medium PEDOT:PSS at high shear rate.

The volume fraction $(\phi)$ of GO into GO/PEDOT solutions was calculated by applying the rule of mixtures starting from the density of the mixtures (Table 1).

Referring to the physical significance of the $\mathrm{k}_{1}$ and $\mathrm{k}_{2}$ parameters introduced in Eq. (3), $\mathrm{k}_{1}$ takes into account the shape of the particles in suspension. Guth found that in the case of non-spherical particles, $\mathrm{k}_{1}$ depends on the aspect ratio [32,33,35], $\mathrm{p}$, according to following equation:

$$
k_{1}=\frac{p}{2 \ln (2 p) 3}+2
$$

$\mathrm{k}_{2}$ was first introduced by Batchelor in order to consider the increase in the viscosity taking place in more concentrated suspensions.

The theoretical predictions obtained by applying this model were in good agreement with the experimental rheological data of Figure 1(a), as shown in Figure 1(b). They also allowed determining 
the following numerical values of the parameters $\mathrm{k}_{1}$ and $\mathrm{k}_{2}: 30.2$ and 119.6 respectively. The mean value of the aspect ratio (p) can be directly estimated by the size distribution of the GO sheets achieved by combining AFM and SEM quantitative analysis and amounting to ca 350 [Liscio A. et al., 2D Materials 2017]. Combining the size analysis and the eq.4, it is also possible to calculate the k1 which amounts to $29 \pm 18$, in good agreement with the value obtained by fit strongly confirming the complete exfoliation of GO sheets in the polymer matrix.

The rheological characterization shows that a good dispersion of the GO filler into PEDOT:PSS was obtained by the solvent swelling method, used in this work. After adding GO to the PEDOT:PSS, the dimension of the filler was still nanometric, also at high GO concentration, and the viscosity of the nanocomposite solutions has reached values still suitable to the film processing by spin coating, which is the standard deposition method used for PEDOT:PSS for photovoltaic application.

In order to confirm the good quality of the GO dispersion, XRD measurements, were carried out on PEDOT:PSS and nanocomposite films, drop casted on a glass substrate.

The X-ray diffraction patterns of the GO filler, reported in our previous papers [16,36], evidence the presence of the characteristic (001) diffraction peak of GO at $2 \theta=11.12^{\circ}$ (black vertical line in Figure 2), corresponding to an interlayer distance of about $0.80 \mathrm{~nm}$. When $\mathrm{GO}$ was incorporated in PEDOT:PSS, the 001 reflection disappeared, confirming the complete loss of the crystalline order perpendicular to the graphitic planes, probably due to the good dispersion of the GO sheet in the polymer matrix, with the intercalation of the polymer chain between GO sheet and the consequent complete GO exfoliation. However, in the presence of $0.25 \%$ wt. of GO, a slight band appears at low degree, attributed to a possible aggregation of GO filler, due to the higher GO concentration.

This latter behavior was also confirmed by SEM analysis performed on the films deposited by spin coating on ITO substrate. $\mathrm{GO}_{0.05}$ PEDOT solution appears as a continuous film with some GO aggregates, meanwhile by increasing GO content up to $0.25 \%$ wt., the presence of the aggregates increases, which can be seen in Figure 3. By using the software of image analysis, Image J, the average diameter of the fillers, calculated from SEM images, was equal to 24 and 35 for 0.05 and 
$0.25 \%$ of GO content, respectively. Higher GO concentration determines the presence of higher GO aggregates, as shown by the previous characterization. However, both XRD and SEM analyses show good and homogeneous GO dispersion into PEDOT:PSS matrix, also at lower GO concentration, confirming that the method used in this work was appropriate for the nanocomposite preparation.

Before the thermal annealing, thermal analysis (DSC/TGA) was carried out on the films in order to better understand the influence of the several concentrations of GO on thermal stability of neat PEDOT:PSS.

DSC analysis shows that all the films exhibit broad endothermic peak between 20 and $160{ }^{\circ} \mathrm{C}$, during the first annealing (Figure 4(a)), which disappears during the second annealing. This indicates that the film releases water during the heating process, as observed by Zhou et al. [29], and is also confirmed by the first weight loss step of TGA curves of the films from $20^{\circ} \mathrm{C}$ to about $155^{\circ} \mathrm{C}$ reported in Figure 4(b) $[29,37]$. The TGA curves of Figure 4(b) evidence a weight loss of $22.7 \%$ for the neat PEDOT:PSS film, which slightly decreases by adding GO to about $18.9 \%$.

Combining DSC and TGA results together provides more refined information about the water loss and the energy associated to their weight loss, allowing to calculate the characteristic energy for dehydration by using the following equation:

$$
E=M\left(\frac{Q}{W}\right)
$$

where, $\mathrm{M}$ is the water molecular weight $\left(18 \mathrm{~g} \mathrm{~mol}^{-1}\right), \Delta \mathrm{Q}$ is the amount of the heat absorbed during the dehydration, estimated from DSC, and $\Delta \mathrm{W}$ is the weight loss of water estimated from TGA. Both thermal analysis were performed in the temperature range between 20 and $155^{\circ} \mathrm{C}$.

The characteristic dehydration energy calculated for all the films is in the range of the energy of moderate hydrogen bonds $\left(10\right.$ to $\left.60 \mathrm{~kJ} \mathrm{~mol}^{-1}\right)$. This suggests that the water molecules are hydrogenbonded with PSS chains in PEDOT:PSS [29] and probably with OH groups in the samples containing GO. However, the characteristic dehydration energy for the PEDOT:PSS decreases from 39 to $33 \mathrm{~kJ}$ $\mathrm{mol}^{-1}$ by adding $0.05 \%$ of GO, while it increases up to 54 and $41 \mathrm{~kJ}$ in the presence of 0.15 and $0.25 \%$ 
of GO respectively, showing a different interaction with water molecules influenced by the presence of the doping element in the PEDOT:PSS.

The exothermic peak, at around $190^{\circ} \mathrm{C}$, which appears in the DSC thermogram of the nanocomposite, in the presence of 0.15 and $0.25 \%$ wt of GO, can be certainly attributed to the removal of labile oxygen functional groups, consequently to the thermal reduction of GO dispersed in the polymer matrix [38]. This peak was not observed in the presence of $0.05 \%$ of GO, probably because the oxygen content groups of GO, lost during the thermal treatment, were very low to be sensed by the instrument. This was confirmed by TGA analysis, indeed it evidences a second weight loss of about $8 \%$ from 155 to $250^{\circ} \mathrm{C}$ for the samples containing 0.15 and $0.25 \%$ of $\mathrm{GO}$, which is not observed for pristine PEDOT:PSS and PEDOT:PSS with $0.05 \%$ of GO.

The third weight loss, observed by TGA analysis, from $250^{\circ} \mathrm{C}$ to about $600^{\circ} \mathrm{C}$, was due to oxidizing decomposition of the skeletal PEDOT and/or PSS backbone chain structure [37]. In particular, at around $250^{\circ} \mathrm{C}$, the PSS sulfonate group fragmentation occurs and over $350^{\circ} \mathrm{C}$, the carbon oxidation is evident [39]. As reported in Table 3, the thermal degradation of PEDOT:PSS became at about $268^{\circ} \mathrm{C}$ up to $510^{\circ} \mathrm{C}$ and causes a weight loss of $36 \%$. By adding $\mathrm{GO}$, the onset point was delayed, in particular in the presence of 0.15 and $0.25 \%$, and the residue was increased, showing that GO influences the thermal stability of PEDOT:PSS. In particular, the possible reduction of GO, caused by temperature, has the potentiality to positively influence the stability of the film by increasing it. This is an important result for the purpose of photovoltaic application.

All the films are thermally stable at $140^{\circ} \mathrm{C}$, so no degradation occurs during annealing treatment. Both the characterizations confirmed that the nanocomposite films were appropriate for the selected application, since the presence of the filler does not affect the temperature of film processing and their thermal stability, rather than increasing it. Therefore, the processing parameters used for the PEDOT:PSS deposition can be used to deposit the nanocomposite films.

The influence of GO doping on the wettability of PEDOT:PSS on ITO was evaluated by contact angle measurements (Figure 5). The addition of GO into PEDOT:PSS reduces the contact angle of 
PEDOT:PSS based solutions on ITO substrate from $93.6^{\circ} \pm 6.2$ to $81.5^{\circ} \pm 0.6$ (Figure $5(\mathrm{a})$ ), ensuring better coverage of substrate. The solution adhesion, in fact, became higher by increasing GO concentration in the PEDOT:PSS, suggesting that the presence of GO improves the interfacial adhesion than the lower substrate compatibility, basic factor to obtain a good layer in a photovoltaic application.

In addition the UV-vis absorption measurements, reported in Figure 6, show that the spectra of PEDOT:PSS does not change by adding GO, after annealing at $140^{\circ} \mathrm{C}$.

\section{CONCLUSION}

The effect of an experimental graphene oxide (GO), prepared by a modified Hummers method, on the thermal and physical properties of $\operatorname{Poly}(3,4$ ethylenedioxythiophene):poly(styrenesulfonate)(PEDOT:PSS), applied on an indium-tin-oxide (ITO) anode, as hole transport layer (HTL) in perovskite solar cells, was investigated as a function of the GO concentration. The viscosity of the GO/PEDOT:PSS mixtures increases by increasing GO content, still remaining appropriate for the spin coating process, conventionally used for the PEDOT:PSS deposition in the specific application and showing, in turn, a good dispersion of the GO filler into the conductive matrix. This latter behavior was also confirmed by XRD analysis, since the GO/PEDOT:PSS pattern evidenced the disappearance of the typical XRD 001 reflection peak of GO, suggesting the complete loss of the crystalline order perpendicular to the graphitic planes. However, due to a possible aggregation of the GO filler, the highest concentration of GO (i.e. $0.25 \%$ wt.) showed a slight band at low degree. This latter result was also evident from SEM analysis, performed on the films deposited by spin coating on ITO substrate. The thermal analysis, performed by TGA and DSC, evidenced a possible reduction of GO, caused by the increased temperature. This is an important result for the purpose of photovoltaic application, since the reduction of GO could improve the stability of the composite film. In addition, the presence of GO in PEDOT:PSS matrix guarantees 
better coverage of the substrate, as evidenced by the reduction of the contact angle of the PEDOT:PSS based solutions on ITO substrate from about $94^{\circ}$ to about $81^{\circ}$, which allows better adhesion of the solution, by increasing GO concentration in the PEDOT:PSS, remaining unchanged the UV vis absorption capability.

\section{ACKNOWLEDGMENTS}

The research leading to these results has received funding from the European Union Horizon 2020 Framework Programme under grant agreement n696656 Graphene Core 1 and the EC Marie-Curie ITN-iSwitch (GA no. 642196).

[1] T. A. Skotheim, (Marcel Dekker, 1986).

[2] J. R. Reynolds, T. A. Skotheim, and R. L. Elsenbaumer, Handbook of conducting polymers (Marcel Dekker, 1998).

[3] T. A. Skotheim and J. Reynolds, Handbook of Conducting Polymers, 2 Volume Set (CRC press, 2007).

[4] J. Ouyang, Displays 34, 423 (2013).

[5] S. Jönsson, J. Birgerson, X. Crispin, G. Greczynski, W. Osikowicz, A. D. Van Der Gon, W. R. Salaneck, and M. Fahlman, Synthetic Metals 139, 1 (2003).

[6] L. Groenendaal, F. Jonas, D. Freitag, H. Pielartzik, and J. R. Reynolds, Advanced Materials 12, $481(2000)$.

[7] Y. Cao, G. Yu, C. Zhang, R. Menon, and A. Heeger, Synthetic Metals 87, 171 (1997).

[8] U. Lang, E. Müller, N. Naujoks, and J. Dual, Advanced Functional Materials 19, 1215 (2009).

[9] A. Mei et al., Science 345, 295 (2014).

[10] N. J. Jeon, J. H. Noh, Y. C. Kim, W. S. Yang, S. Ryu, and S. I. Seok, Nature materials 13, 897 (2014).

[11] N. J. Jeon, J. H. Noh, W. S. Yang, Y. C. Kim, S. Ryu, J. Seo, and S. I. Seok, Nature 517, 476 (2015).

[12] T. Liu, D. Kim, H. Han, A. R. bin Mohd Yusoff, and J. Jang, Nanoscale 7, 10708 (2015).

[13] A. Fallahi, M. Alahbakhshi, E. Mohajerani, F. Afshar Taromi, A. R. Mohebbi, and M.

Shahinpoor, The Journal of Physical Chemistry C 119, 13144 (2015).

[14] S. I. Ahn, K. Kim, J. R. Jung, K. Y. Kang, S. M. Lee, J. Y. Han, and K. C. Choi, Chemical Physics Letters 625, 36 (2015).

[15] A. Giuri et al., IEEE Transactions on Nanotechnology PP (2016).

[16] A. Giuri et al., Advanced Functional Materials 26, 6985 (2016).

[17] K. Sun, Y. Xia, and J. Ouyang, Solar energy materials and solar cells 97, 89 (2012).

[18] S. Pei and H.-M. Cheng, Carbon 50, 3210 (2012).

[19] M. Hirata, T. Gotou, S. Horiuchi, M. Fujiwara, and M. Ohba, Carbon 42, 2929 (2004).

[20] F. Perrozzi et al., The Journal of Physical Chemistry C 117, 620 (2012). 
[21] H. S. Dehsari, E. K. Shalamzari, J. N. Gavgani, F. A. Taromi, and S. Ghanbary, RSC Advances 4, 55067 (2014).

[22] Y. H. Ding, P. Zhang, Q. Zhuo, H. M. Ren, Z. M. Yang, and Y. Jiang, Nanotechnology 22, 215601 (2011).

[23] A. Giuri et al., Science of Advanced Materials 7, 2445 (2015).

[24] S. Rella, A. Giuri, C. E. Corcione, M. R. Acocella, S. Colella, G. Guerra, A. Listorti, A. Rizzo, and C. Malitesta, Vacuum 119, 159 (2015).

[25] S. G. Chengzhou Zhu, Youxing Fang, and Shaojun Dong*, ACS Nano 4, 2429 (2010).

[26] S. Stankovich, D. A. Dikin, R. D. Piner, K. A. Kohlhaas, A. Kleinhammes, Y. Jia, Y. Wu, S. T. Nguyen, and R. S. Ruoff, Carbon 45, 1558 (2007).

[27] J. Zhou, D. H. Anjum, G. Lubineau, E. Q. Li, and S. T. Thoroddsen, Macromolecules 48, 5688 (2015).

[28] M. Mauro, M. R. Acocella, C. E. Corcione, A. Maffezzoli, and G. Guerra, Polymer 55, 5612 (2014).

[29] J. Zhou, D. H. Anjum, L. Chen, X. Xu, I. A. Ventura, L. Jiang, and G. Lubineau, J. Mater.

Chem. C 2, 9903 (2014).

[30] M. M. Cross, Journal of colloid science 20, 417 (1965).

[31] G. Batchelor, Journal of Fluid Mechanics 83, 97 (1977).

[32] C. E. Corcione, F. Freuli, and A. Maffezzoli, Polymer Engineering \& Science 53, 531 (2013).

[33] C. Esposito Corcione, G. Mensitieri, and A. Maffezzoli, Polymer Engineering \& Science 49, 1708 (2009).

[34] A. Einstein, Phys 19, 289 (1906).

[35] C. E. Corcione, A. Cavallo, E. Pesce, A. Greco, and A. Maffezzoli, Polymer Engineering \& Science 51, 1280 (2011).

[36] A. Giuri, S. Colella, A. Listorti, A. Rizzo, and C. E. Corcione, in VIII INTERNATIONAL CONFERENCE ON "TIMES OF POLYMERS AND COMPOSITES": From Aerospace to Nanotechnology (AIP Publishing, 2016), p. 020115.

[37] Y. Xu, Y. Wang, J. Liang, Y. Huang, Y. Ma, X. Wan, and Y. Chen, Nano Research 2, 343 (2009).

[38] S. Park, J. An, J. R. Potts, A. Velamakanni, S. Murali, and R. S. Ruoff, Carbon 49, 3019 (2011).

[39] A. Elschner, S. Kirchmeyer, W. Lovenich, U. Merker, and K. Reuter, PEDOT: principles and applications of an intrinsically conductive polymer (CRC Press, 2010). 